\title{
Comparison of the influence of three fibroid treatment options: supracervical hysterectomy, ulipristal acetate and uterine artery embolization on ovarian reserve - an observational study
}

\author{
Piotr Czuczwar, Anna Stepniak (iD, Pawel Milart, Tomasz Paszkowski and Slawomir Wozniak
}

\begin{abstract}
Background: To assess and compare the influence of three fibroid treatment options: supracervical hysterectomy, ulipristal acetate and uterine artery embolization on ovarian reserve.

Methods: Prospective, observational, open-label study performed at the 3rd Chair and Department of Gynecology of the Medical University of Lublin, Poland. Premenopausal Caucasian women with symptomatic uterine fibroids were recruited into 3 groupspatients qualified for supracervical hysterectomies; patients qualified for preoperative ulipristal acetate (UPA) treatment scheduled for supracervical hysterectomies or myomectomies; patients qualified for uterine artery embolization (UAE). The following markers of ovarian reserve were investigated: antral follicle count (AFC), anti-Mullerian hormone (AMH), inhibin B (INHB), follicle stimulating hormone (FSH) and estradiol (E2). These markers were assessed before and 3 months after supracervical hysterectomies, before and 3 months after UAEs, and before and after 3 months of UPA treatment, before the scheduled surgeries. Baseline characteristics (age, parity, dominant fibroid volume, hemoglobin level, BMI, as well as AFC, AMH, INHB, FSH and E2) were compared between the study groups by Kruskall-Wallis ANOVA. Pre- and post-interventional values of AFC, $\mathrm{AMH}, \mathrm{INHB}, \mathrm{FSH}$ and E2 in the studied groups were compared with the Wilcoxon matched pairs test.

Results: Twenty-six, 27 and 30 patients were included in the final analysis in the supracervical hysterectomy, UPA and UAE groups, respectively. Three months after supracervical hysterectomy INHB and E2 significantly decreased, while AFC, AMH and FSH remained unchanged. After 3 months of UPA treatment the values of all the assessed markers of ovarian reserve were not significantly different in comparison to baseline. Conversely, three months after UAE the values of AFC, AMH, INHB, and E2 were significantly decreased, while FSH was significantly increased.

Conclusions: Of the compared fibroid treatment methods UAE seems to have the greatest impact on ovarian function and should not be offered to patients concerned about their ovarian function. Supracervical hysterectomy did not affect the most accurate markers of ovarian reserve, and therefore appears to be safe in terms of ovarian function. UPA did not change any of the studied markers of ovarian reserve and seems a reasonable option when ovarian function is concerned.
\end{abstract}

\footnotetext{
* Correspondence: aanna.stepniak@gmail.com

3rd Department of Gynecology of the Medical University of Lublin, ul.

Jaczewskiego 8, 20-954 Lublin, Poland
}

(c) The Author(s). 2018 Open Access This article is distributed under the terms of the Creative Commons Attribution 4.0 International License (http://creativecommons.org/licenses/by/4.0/), which permits unrestricted use, distribution, and reproduction in any medium, provided you give appropriate credit to the original author(s) and the source, provide a link to the Creative Commons license, and indicate if changes were made. The Creative Commons Public Domain Dedication waiver (http://creativecommons.org/publicdomain/zero/1.0/) applies to the data made available in this article, unless otherwise stated. 


\section{Background}

Surgical management constitutes the majority of fibroid treatment, but minimally-invasive alternatives are also available [1]. Uterine artery embolization (UAE) is a procedure, during which embolic material is administered into the uterine arteries to decrease the blood supply of fibroids [2]. It has previously been shown, that UAE results in reduction of fibroid symptoms and improvement of the quality of life [2]. Recent studies have demonstrated the efficacy of ulipristal acetate (UPA), a selective progesterone receptor modulator (SPRM), in the medical management of fibroids [3].

Many women seek alternative treatment options for fibroids because of their concern about future fertility, and, interestingly, irrespective of the desire for childbearing [4]. Premature menopause may have significant negative impact on the future quality of life and morbidity, such as increased risk of osteoporosis, cardiovascular disease, and all-cause mortality [5].

The impact of various fibroid treatment options on ovarian function has been debated [6]. The influence of fibroid surgery and UAE on ovarian reserve has previously been investigated in numerous studies but the possible decline of ovarian reserve after these interventions remains controversial [7]. Available data on the effects of SPRMs on ovarian reserve are scarce $[8,9]$. The aim of this study was to investigate the influence of three fibroid treatment methods (supracervical hysterectomy, UAE and UPA) on ovarian reserve.

\section{Methods}

This prospective observational open-label single center study was performed at the 3rd Chair and Department of Gynecology of the Medical University of Lublin, Poland. Patients were recruited from December 2012 to November 2013 and followed-up for 3 months. Three groups of patients with symptomatic fibroids were recruited: patients qualified for supracervical hysterectomies with bilateral salpingectomy; patients qualified for preoperative UPA treatment before supracervical hysterectomies or myomectomies; patients qualified for UAE. Due to the capacity of biochemical kits and the expected patient numbers the number of patients was restricted to 29, 29 and 30 in the respective groups. Because UPA patients were scheduled to undergo surgeries 3 months after the beginning of UPA treatment, follow-up was limited to 3 months in all groups. It must be emphasized, that even though patients receiving UPA underwent surgeries after the treatment, the assessment of ovarian reserve in the UPA group was performed before the surgical interventions, because the objective of this study was to assess the possible impact of UPA on ovarian reserve, and not of both
UPA and surgical treatment. Inclusion criteria were: symptomatic uterine fibroids, abnormal uterine bleeding, dominant fibroid diameter of 30-80 mm, intramural location of fibroids, normal ovarian reserve (anti-Mullerian hormone $(\mathrm{AMH})(>1.0 \mathrm{ng} / \mathrm{ml})$ and follicle stimulating hormone $(\mathrm{FSH})(<20 \mathrm{mIU} / \mathrm{ml})$ on days $2-4$ of the menstrual cycle and regular menstrual bleeding), no history of ovarian pathology, normal endometrial biopsy, normal endometrial appearance on ultrasound (according to current guidelines [10]) and no previous hormonal treatment. Baseline characteristics (age, parity, dominant fibroid volume, hemoglobin level, BMI) were recorded in all patients. Fibroid volume was estimated by transvaginal ultrasound using the ellipsoid formula.

In patients receiving UPA therapy (Esmya $\odot$, Gedeon Richter, Budapest, Hungary), $5 \mathrm{mg} / 24 \mathrm{~h}$ of UPA was administered orally during the first 4 days of menstrual bleeding and continued for 3 months. Indications for UPA therapy included a planned myomectomy/hysterectomy because of abnormal uterine bleeding, anemia and pelvic pain. Patients were qualified for UAE according to the Clinical Practice Guidelines of the Society of Obstetricians and Gynaecologists of Canada [11, 12]. During UAE polyvinyl alcohol particles were administered until complete stasis of contrast had been achieved in both uterine arteries. Only patients with successful bilateral embolization of the uterine arteries were included in the study. Written informed consent was obtained from all participants and the study protocol was accepted by the local bioethics committee. This study was performed according to the STROBE Statement Checklist for observational studies.

The following markers of ovarian reserve were investigated: antral follicle count (AFC), AMH, inhibin B (INHB), FSH and E2. AFC was measured according to current guidelines [13] between the 2-4 day of the cycle using a Samsung Medison V20 Prestige (Samsung Medison, Seoul, South Korea) equipped with a transvaginal probe. All antral follicles of $2-10 \mathrm{~mm}$ in diameter were included. AFC > 10 was considered to be normal. To determine the time for follow-up assessment after supracervical hysterectomies, the patients were asked to perform ovulation tests and/or present for ultrasound monitoring of the cycle.

FSH, E2, AMH and INHB levels were measured in plasma samples collected between the 2-4 day of the cycle by commercially available Enzyme-linked Immunosorbent Assay Kits according to the manufacturer's instructions. The following kits were used: E90228Hu for AMH (Uscn Life Science Inc., Wuhan, Hubei, China); E90760Hu for INHB (Uscn Life Science Inc., Wuhan, Hubei, China); FR E-2400 for FSH and FR E-2000 for E2 (Labor Diagnostika Nord GmBH \& Co. KG, Nordhorn, Germany). The following cut-points for normal values 
were used: $<20 \mathrm{mIU} / \mathrm{ml}$ for $\mathrm{FSH} ;>50 \mathrm{pg} / \mathrm{ml}$ for E2; $>1 \mathrm{ng} / \mathrm{ml}$ for $\mathrm{AMH}$; and $>10 \mathrm{pg} / \mathrm{ml}$ for INHB.

\section{Statistics}

Statistical analysis was performed using Statistica 12 (Statsoft, Tulsa, OK, USA). The normality of continuous variables was tested with the Shapiro-Wilk test. Baseline characteristics (age, parity, dominant fibroid volume, hemoglobin level, BMI, as well as AFC, AMH, INHB, FSH and E2) were compared between the study groups by Kruskal-Wallis ANOVA. Pre- and post-interventional values of AFC, AMH, INHB, FSH and E2 in the studied groups were compared with the Wilcoxon matched pairs test. To confront the impact of the studied treatment options on AFC, AMH, INHB, FSH and E2, percentage changes of these parameters were calculated for each study group and compared by Kruskal-Wallis ANOVA with post-hoc analysis. Percentages of cases with abnormal post-interventional values of AFC, AMH, INHB, FSH and E2 were compared between the studied groups with the Chi square test. $P$ values $<0.05$ were considered significant.

\section{Results}

\section{Participants}

Flow-chart of the study design is shown on Fig. 1. Forty-two patients qualified for supracervical hysterectomies with bilateral salpingectomies, 35 qualified for preoperative UPA treatment and 38 qualified for UAE were screened to obtain the required number of patients in the study groups $(29,29$ and 30 respectively). After performing the biochemical tests at the end of the study 3 patients were excluded from the supracervical hysterectomy group and 2 from the UPA group due to abnormal baseline AMH levels, leaving 26, 27 and 30 patients in the study groups, respectively.

Patient demographic characteristics are shown in Table 1. Age, parity, dominant fibroid volume, hemoglobin levels and BMI were not significantly different between the studied groups. Similarly, baseline AFC values and plasma levels of AMH, INHB, FSH and E2 were not significantly different.

\section{Main results}

Pre- and post-interventional AFC values and plasma levels of AMH, INHB, FSH and E2 are shown in Table 2. Three months after supracervical hysterectomy INHB and E2 significantly decreased, while AFC, AMH and FSH remained unchanged. After 3 months of UPA treatment the values of all the assessed markers of ovarian reserve were not significantly different in comparison to baseline. Conversely, three months after UAE the values of AFC, AMH, INHB, and E2 were significantly decreased, while FSH was significantly increased. Percentage changes of the investigated markers of ovarian reserve are shown in Table 3. In all studied markers of ovarian reserve percentage changes at 3-month follow-up were significantly greater after UAE than after supracervical hysterectomy or UPA. There were no significant differences in percentage changes of ovarian reserve markers between the supracervical hysterectomy and UPA groups (Table 3). The percentage of cases with abnormal post-interventional values at 3-month follow-up in the supracervical hysterectomy, UPA and UAE groups was 19.2; 18.5 and $56.7 \%$ for $\operatorname{AFC}(p=0.006), 3.9 ; 3.7$ and $36.7 \%$ for $\mathrm{AMH}$ $(p<0.001), 15.4 ; 18.5$ and $46.7 \%$ for INHB $(p=0.014)$, $11.5 ; 18.5$ and $43.4 \%$ for FSH $(p=0.015)$ and 0 ; 0 and $43.3 \%$ for $\mathrm{E} 2(p<0.001)$, respectively.

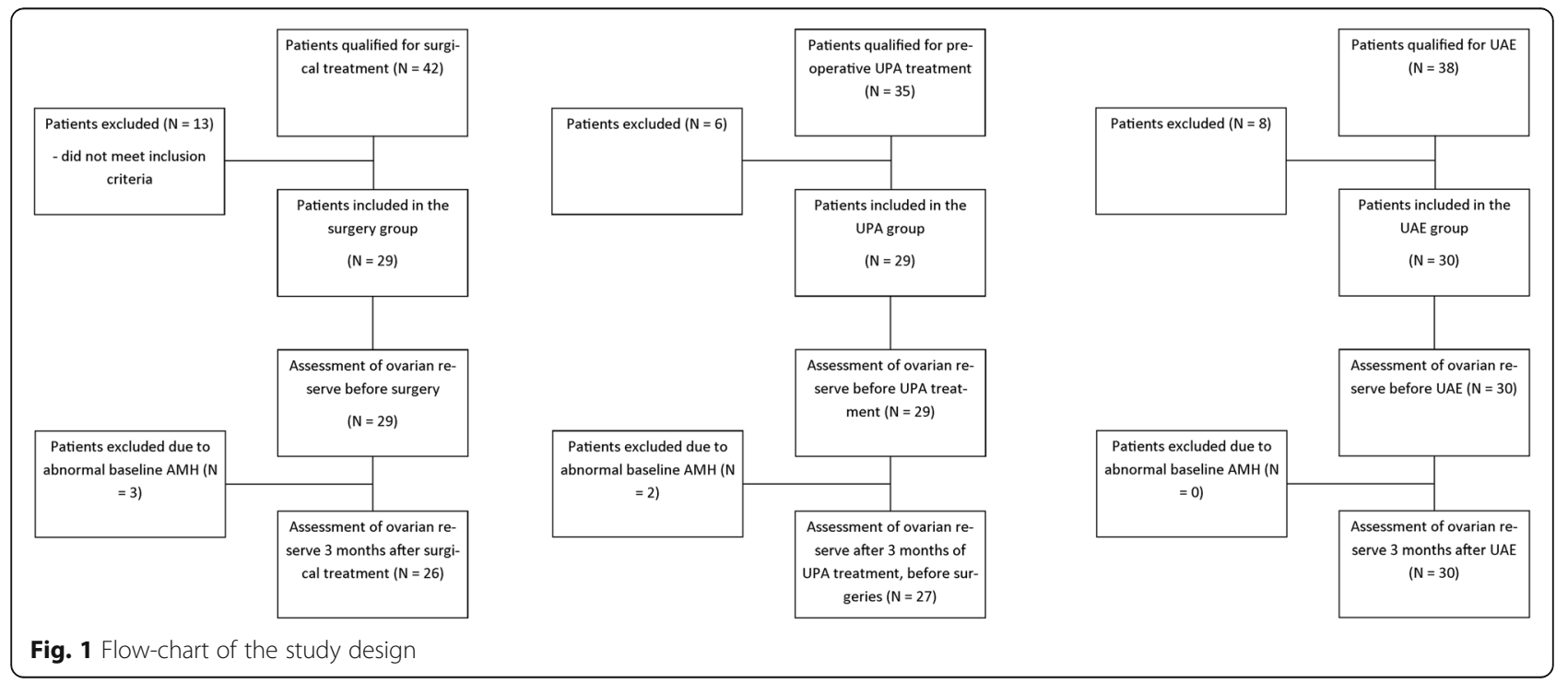


Table 1 Baseline characteristics of the patients qualified for hysterectomy, ulipristal acetate treatment or uterine artery embolization

\begin{tabular}{lllll}
\hline & Supracervical hysterectomy & UPA & UAE & P \\
\hline Number of patients & 26 & 27 & 30 & $35(33-40)$ \\
Age & $38(36-43)$ & $37(34-43)$ & $1(1-2)$ & N/A \\
Parity & $2(1-2)$ & $2(1-2)$ & 10.05 \\
Dominant fibroid Volume $\left(\mathrm{cm}^{3}\right)$ & $103.3(87.6-127.6)$ & $104.6(85.2-132.1)$ & $107.75(87.4-131.1)$ & $>0.05$ \\
Hemoglobin $(\mathrm{g} / \mathrm{dl})$ & $10.5(9.0-11.9)$ & $10.2(8.9-11.1)$ & $10.3(9.1-11.4)$ & 0.05 \\
BMI $\left(\mathrm{kg} / \mathrm{m}^{2}\right)$ & $27.5(23.6-31.3)$ & $28.1(24.1-32.9)$ & $26.9(22.5-30.6)$ & $>0.05$ \\
\hline
\end{tabular}

Data are presented as median (interquartile range). N/A not applicable, UPA ulipristal acetate, UAE uterine artery embolization

At 3-month follow-up, abnormal uterine bleeding had ceased in all cases in the UPA and UAE groups and no significant side-effects were observed. In the surgery group all patients underwent supracervical hysterectomies with bilateral salpingectomies, both ovaries were spared in all cases, no complications in the post-operative course were noted.

\section{Discussion}

The main finding of this study is that UAE had the greatest impact on ovarian reserve. Supracervical hysterectomy affected only INHB and E2 levels, while no significant changes in AFC, AMH and FSH were observed. UPA did not affect any of the investigated markers of ovarian reserve. Percentage changes of the investigated parameters were significantly greater in the UAE group than in the UPA and hysterectomy groups. Similarly, the

Table 2 Parameters assessed before and 3 months after hysterectomy, ulipristal acetate therapy or uterine artery embolization

\begin{tabular}{llll}
\hline & Baseline & 3 months follow-up & $P$ \\
\hline AFC Hysterectomy & $14(12-24)$ & $14(9-19)$ & $>0.05$ \\
AFC UPA & $15(13-24)$ & $15(12-19)$ & $>0.05$ \\
AFC UAE & $19(15-24)$ & $8(4-12)$ & $<0.001$ \\
AMH Hysterectomy & $2.64(2.03-3.84)$ & $2.66(1.91-3.67)$ & $>0.05$ \\
AMH UPA & $2.89(2.11-4.34)$ & $2.91(2.22-4.12)$ & $>0.05$ \\
AMH UAE & $3.04(2.53-3.7)$ & $1.28(0.49-2.11)$ & $<0.001$ \\
INHB Hysterectomy & $28.09(20.76-35.33)$ & $24.05(18.79-32.74)$ & $<0.001$ \\
INHB UPA & $25.16(14.78-47.34)$ & $26.78(13.78-42.33)$ & $>0.05$ \\
INHB UAE & $25.11(15.67-33.9)$ & $10.78(4.71-15.83)$ & $<0.001$ \\
FSH Hysterectomy & $8.32(5.51-13.47)$ & $9.6(5.62-12.59)$ & $>0.05$ \\
FSH UPA & $11.21(4.56-16.78)$ & $9.88(6.12-19.34)$ & $>0.05$ \\
FSH UAE & $8.75(6.3-11.12)$ & $16.99(12.35-41.33)$ & $<0.001$ \\
E2 Hysterectomy & $81.85(73.62-108.12)$ & $74.59(59.34-115.43)$ & 0.005 \\
E2 UPA & $90.23(78.34-136.45)$ & $94.32(67.22-154.33)$ & $>0.05$ \\
E2 UAE & $98.44(87.32-115.89)$ & $57.37(39.34-93.22)$ & $<0.001$
\end{tabular}

Data are presented as median (interquartile range). $A F C$ antral follicle count, $A M H$ anti-Mullerian hormone, $\mathrm{ng} / \mathrm{ml}, I N H B$ inhibin $\mathrm{B}, \mathrm{pg} / \mathrm{ml}, F S H$ follicle stimulating hormone, $\mathrm{mlU} / \mathrm{ml}, E 2$ estradiol, $\mathrm{pg} / \mathrm{ml}$, UPA ulipristal acetate, UAE uterine artery embolization percentage of patients with abnormal post-interventional ovarian reserve markers was the greatest in the UAE group.

Ovarian reserve is understood as the number and quality of remaining oocytes and describes individual reproductive potential. There is no uniformly accepted definition of decreased ovarian reserve [14]. The following tests are used to assess ovarian reserve: FSH, E2, INHB, AMH and the clomiphene citrate challenge test as well as AFC and ovarian volume. AMH is accepted to be the most accurate test [14].

The possible mechanism of compromising ovarian reserve seems to be the clearest for hysterectomies. Three hypotheses are considered [6]. Transecting the utero-ovarian ligament with the ovarian branch of the uterine artery decreases the blood supply of the ovary and may lead to ischemia and loss of primordial follicles [15]. The underlying indication for the hysterectomy, such as fibroids or abnormal uterine bleeding, may also have a significant impact on ovarian reserve [16]. Finally, it has been speculated that the uterus might secrete paracrine factors regulating the recruitment of follicles, the loss of which could possibly diminish ovarian reserve [6]. For UAE it seems most likely that the embolic material may reach the ovary and block the ovarian microvasculature resulting in minor ischemia and reduction of the primordial follicular pool [6]. Similarly as in the case of hysterectomies, the presence of fibroids may be responsible for the lower ovarian reserve in general [6]. For UPA, this subject has not been debated.

The influence of UAE on ovarian reserve has been previously investigated but remains controversial. In a randomized trial by Mara et al. comparing the outcomes of UAE and myomectomy in fibroid patients, 6 months after the procedure significantly more patients after UAE had elevated FSH levels [17]. The randomized EMMY trial compared FSH and AMH levels after hysterectomy and UAE in premenopausal women with symptomatic fibroids within a 2-year follow-up. The risk of ovarian failure (defined as an increase in $\mathrm{FSH}>40 \mathrm{mIU} / \mathrm{ml}$ ) was similar in both the hysterectomy and UAE groups. However, only in the UAE group a significant decrease in AMH levels 
Table 3 Median percentage changes in the investigated markers of ovarian reserve 3 months after supracervical hysterectomy, uterine artery embolization or after a course of UPA therapy

\begin{tabular}{lllll}
\hline Variable & Study group & Percentage change at 3-month follow-up & $P$ & Post-hoc analysis \\
\hline AFC & Hysterectomy & $-8.4(-15--4.2)$ & $<0.001$ & Hysterectomy vs UPA: NS \\
& UPA & $-7.8(-14.3-0)$ & Hysterectomy vs UAE: $p<0.001$ \\
& UAE & $-63.4(-78.9--37.5)$ & UPA vs UAE: $p<0.001$ \\
AMH & Hysterectomy & $-3.5(-10-4.8)$ & $<0.001$ & Hysterectomy vs UPA: NS \\
& UPA & $-5.8(-7.7--5.1)$ & Hysterectomy vs UAE: $p<0.001$ \\
& UAE & $-61.9(-82--47.4)$ & UPA vs UAE: $p<0.001$ \\
INH & Hysterectomy & $-7.4(-15.1--2.5)$ & Hysterectomy vs UPA: NS \\
& UPA & $-7.0(-10.6-3.0)$ & Hysterectomy vs UAE: $p<0.001$ \\
& UAE & $-60.7(-74.5--51.2)$ & UPA vs UAE: $p<0.001$ \\
Hysterectomy & $21.5(-9.1-35)$ & Hysterectomy vs UPA: NS \\
& UPA & $12.7(-9.8-27)$ & Hysterectomy vs UAE: $p<0.001$ \\
& UAE & $125.6(51-295.8)$ & & UPA vs UAE: $p<0.001$ \\
& Hysterectomy & $-13.3(-19.5--3.5)$ & & Hysterectomy vs UPA: NS \\
& UPA & $-3.3(-10.3-11.4)$ & Hysterectomy vs UAE: $p<0.001$ \\
& UAE & $-41(-53.5-7.6)$ & & UPA vs UAE: $p<0.001$ \\
\hline
\end{tabular}

Data are presented as median (interquartile range). $A F C$ antral follicle count, $A M H$ anti-Mullerian hormone, INHB inhibin $\mathrm{B}$, FSH follicle stimulating hormone, E2 estradiol, UPA ulipristal acetate, UAE uterine artery embolization

was observed throughout the study period [18]. In a recently published update of the EMMY trial the authors extended the follow-up to 10 years and reported, that in both groups a similar percentage of patients underwent menopause [19]. The authors concluded that both hysterectomy and UAE affect ovarian reserve. Conversely, Tropeano et al. did not observe significant changes in FSH, AFC and ovarian volume for 5 years after UAE [20]. This difference might be because the women in the EMMY trial were on average 10 years older than in the Tropeano et al. study and younger age may have a protective effect on ovarian function after UAE [6]. Some authors suggested that the impact of UAE on the hormonal markers of ovarian reserve may be transient. Tsikouras et al. reported a significant increase of FSH after UAE, that reached its maximum value at 3 months and returned to baseline values 12 months after the procedure [21]. The authors also investigated changes in AMH levels and reported significant decreases 1 month after UAE in younger patients ( $\leq 45$ years) and 1 and 3 months after UAE in older patients ( $>45$ years). Kim et al. showed a significant decrease of AMH and AFC at 3 and 12 months after UAE [22]. AMH levels remained low after 12 months of follow-up compared to the expected $\mathrm{AMH}$ levels. Interestingly, in patients $<40$ years-old a significant recovery of $\mathrm{AMH}$ between the 3 and 12 months measurements was observed, again suggesting that the ovaries in younger women may be more likely to recover after UAE [22]. Basing on our results and the literature data it seems that there is at least a short-term effect of UAE on ovarian reserve and this treatment option should not be offered to patients concerned about their ovarian function.

Surprisingly, we did not observe significant changes in $\mathrm{AMH}, \mathrm{AFC}$ and FSH after supracervical hysterectomies. Two large prospective cohort studies investigating the association between hysterectomy and menopause were published: the Farquhar et al. and the PROOF study $[16,23]$. Both studies concluded, that hysterectomy is associated with an earlier onset of menopause. However, both studies used only FSH as a marker of ovarian function and the occurrence of menopause as an endpoint. Moreover, it was not clear whether the surgical intervention or the indication for surgery contributed to the earlier onset of menopause [6]. Nevertheless, this appears to be in contradiction with our results. However, in the abovementioned studies many patients underwent unilateral oophorectomies during the surgeries. Moreover, an update of the PROOF trial has been recently published [24]. The authors compared $\mathrm{AMH}$ levels in women undergoing hysterectomies with the control group 1-year after the surgeries. In general, patients undergoing hysterectomies had lower AMH levels compared with the control group. Surprisingly, these differences were not seen among white women, but remained significant in black women [24]. This is in concordance with the results of our study, where only white women were included. Interestingly, the EMMY trial, in which hysterectomy did not exert a permanent effect on AMH levels, also included mainly white women [18]. There is some data suggesting that 
reproductive function differs by race. It has been shown that AMH levels were significantly lower in black women than in white [25]. It may be speculated that white race is a protective factor against the negative effect of hysterectomy on ovarian reserve.

In our study UPA did not affect any of the markers of ovarian reserve. Two previous studies assessed ovarian function after UPA treatment. UPA (up to $10 \mathrm{mg}$ daily) did not affect the levels of E2 and FSH and follicular growth on ultrasound after 84 days of treatment [8]. In a study assessing the long-term efficacy and safety of UPA (four 12-week courses) E2 levels remained stable [9]. Even though E2 and FSH are not reliable markers of ovarian reserve, these results support the statement, that UPA treatment does not affect ovarian reserve.

Most of novel treatment algorithms for fibroids begin with UPA treatment [26] and therefore the influence of this treatment on ovarian reserve could play an important role, especially in patients wishing to conceive. Taking into consideration that an increasing number of pregnancies after UPA treatment is being reported [27], our observation that UPA treatment does not affect the markers of ovarian reserve, further supports the role of UPA in the treatment of patients wishing to preserve fertility.

The strength of our study is that it is the first prospective trial comparing the impact of 3 different fibroid treatment options on ovarian function, in a homogenous population. Many factors were suggested to influence the outcome of fibroid treatment, especially in case of UAE. These include fibroid features such as volume, vascularity and location, but also patient characteristics such as age and menopausal status [28]. Even factors such as patient race may completely change the impact of hysterectomy on ovarian reserve [24]. Therefore, it is difficult to compare the outcomes of various fibroid treatment options observed in different populations. However, our results may not be generalized to other populations.

Our study has some limitations. The main limitation is the short follow-up. During the data collection for this study, UPA was registered only for a single 3-month course of preoperative treatment, which limited the follow-up duration. Recently, the safety of up to 8 consecutive 3 -month UPA courses has been showed [29], which encourages to assess the impact of extended UPA treatment on ovarian function. It is difficult to state, whether the observed reduction of AMH levels 3 months after UAE is maintained over a longer time period. As previously discussed, some studies suggested that the decrease of $\mathrm{AMH}$ at 1-3 months after UAE is transient and recovers within a year, at least in younger patients [20-22]. Other studies showed that this phenomenon may be prolonged (up to 2 years follow-up), especially in older patients $[18,22]$. Further studies are necessary to fully elucidate this issue and identify the possible factors influencing ovarian function after UAE. Our study is relatively small and open-label rather than randomized, therefore some differences in the baseline characteristics of the studied groups that could have affected the final results might not have been identified. However, most of the patients presenting for fibroid treatment had their treatment preference and we decided that it would be more ethical to take it into consideration and make shared decisions with the patients.

\section{Conclusions}

In conclusion, UAE seems to have the greatest impact on ovarian function and should not be offered to patients concerned about their ovarian function. Supracervical hysterectomy did not affect the most accurate markers of ovarian reserve and appears to be safe in terms of ovarian function. UPA did not change any of the studied markers of ovarian reserve and seems a reasonable option when ovarian function is concerned.

\section{Acknowledgements}

This study was supported by a grant from the Medical University of Lublin. No external funding was received for this study.

\section{Funding}

This study was supported by a grant from the Medical University of Lublin, Poland (grant number: DS 329).

\section{Availability of data and materials}

The datasets used during the current study are available from the corresponding author on reasonable request.

\section{Authors' contributions}

PC collected the clinical data, wrote the manuscript. AS prepared database and statistical analysis. PM and TP participated in the treatment and reviewed the manuscript. SW collected the clinical data and revised the manuscript. All authors read and approved the final manuscript.

Ethics approval and consent to participate

This work has been approved by the ethics committee of Medical University of Lublin, and informed consent for publication was obtained from all patients.

Consent for publication

Informed consent for publication was obtained from all patients.

\section{Competing interests}

The authors declare that they have no competing interests.

\section{Publisher's Note}

Springer Nature remains neutral with regard to jurisdictional claims in published maps and institutional affiliations.

Received: 22 February 2018 Accepted: 23 May 2018

Published online: 01 June 2018

\section{References}

1. Matchar DB, Myers ER, Barber MW, Couchman GM, Datta S, Gray RN, et al. Management of uterine fibroids. Evid Rep Technol Assess. 2001;34

2. Tropeano G, Amoroso S, Scambia G. Non-surgical management of uterine fibroids. Hum Reprod Update. 14(3) 
3. Donnez J, Donnez O, Dolmans MM. With the advent of selective progesterone receptor modulators, what is the place of myoma surgery in current practice? Fertil Steril. 2014;102

4. Borah BJ, Nicholson WK, Bradley L, Stewart EA. The impact of uterine leiomyomas: a national survey of affected women. Am J Obstet Gynecol. 2013;209:319.

5. Mondul AM, Rodriguez C, Jacobs EJ, Calle EE. Age at natural menopause and cause-specific mortality. Am J Epidemiol. 2005;162

6. Jacob GP, Oraif A, Power S. When helping hurts: the effect of surgical interventions on ovarian reserve. Hum Fertil. 2016:19

7. Iwase A, Nakamura T, Nakahara T, Goto M, Kikkawa F. Assessment of ovarian reserve using anti-Müllerian hormone levels in benign gynecologic conditions and surgical interventions: a systematic narrative review. Reprod Biol Endocrinol. 2014;12:125.

8. Chabbert-Buffet N, Pintiaux-Kairis A, Bouchard P. Effects of the progesterone receptor modulator VA2914 in a continuous low dose on the hypothalamicpituitary-ovarian axis and endometrium in normal women: a prospective, randomized, placebo-controlled trial. J Clin Endocrinol Metab. 2007;92

9. Donnez J, Donnez O, Matule D, Ahrendt HJ, Hudecek R, Zatik J, et al. Longterm medical management of uterine fibroids with ulipristal acetate. Fertil Steril. 2016;105

10. Singh S, Best C, Dunn S, Leyland N, Wolfmann WL. Abnormal uterine bleeding in women. JOGC. 2013;35

11. SOGC clinical practice guidelines. Uterine fibroid embolization (UFE). Number 150, October 2004. Int J Gynaecol Obstet. 2005;89

12. Carranza-Mamane B, Havelock J, Hemmings R. The Management of Uterine Fibroids in women with otherwise unexplained infertility. Clinical, Sogc Guideline, Practice J Obs Gynaecol Can. 2015;37

13. Broekmans FJM, De Ziegler D, Howles CM, Gougeon A, Trew G, Olivennes F. The antral follicle count: practical recommendations for better standardization. Fertil Steril. 2010;94

14. Practice Committee of the American Society for Reproductive Medicine. Testing and interpreting measures of ovarian reserve: a committee opinion. Fertil Steril. 2012;98

15. Fenton A, Panay N. Does routine gynecological surgery contribute to an early menopause? Climacteric. 2012;15

16. Moorman PG, Myers ER, Schildkraut JM, Iversen ES, Wang F, Warren N. Effect of hysterectomy with ovarian preservation on ovarian function. Obstet Gynecol. 2011;118

17. Mara M, Maskova J, Fucikova Z, Kuzel D, Belsan T, Sosna O. Midterm clinical and first reproductive results of a randomized controlled trial comparing uterine fibroid embolization and myomectomy. Cardiovasc Intervent Radiol. 2008;31

18. Hehenkamp WJ, Volkers NA, Broekmans FJ, de Jong FH, Themmen APN,

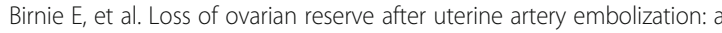
randomised comparison with hysterectomy. Hum Reprod. 2007;22

19. de Bruijn AM, Ankum WM, Reekers JA, Birnie E, van der Kooij SM, Volkers $N A$, et al. Uterine artery embolization vs hysterectomy in the treatment of symptomatic uterine fibroids: 10-year outcomes from the randomized EMMY trial. Am J Obstet Gynecol. 2016;215:745.

20. Tropeano G, Di Stasi C, Amoroso S, Gualano MR, Bonomo L, Scambia G. Long-term effects of uterine fibroid embolization on ovarian reserve: a prospective cohort study. Fertil Steril. 2010;94

21. Tsikouras P, Manav B, Koukouli Z, Trypsiannis G, Galazios G, Souftas D, et al. Ovarian reserve after fibroid embolization in premenopausal women. Minim Invasive Ther Allied Technol. 2017;

22. Kim C-W, Shim HS, Jang H, Song YG. The effects of uterine artery embolization on ovarian reserve. Eur J Obstet Gynecol Reprod Biol. 2016;206

23. Farquhar CM, Sadler L, Harvey SA, Stewart AW. The association of hysterectomy and menopause: a prospective cohort study. BJOG. 2005;112

24. Trabuco EC, Moorman PG, Algeciras-Schimnich A, Weaver AL, Cliby WA Association of Ovary-Sparing Hysterectomy with Ovarian Reserve. Obste Gynecol. 2016;127

25. Seifer DB, Golub ET, Lambert-Messerlian G, Benning L, Anastos K, Watts DH, et al. Variations in serum müllerian inhibiting substance between white, black, and Hispanic women. Fertil Steril. 2009;92

26. Donnez J, Dolmans M-M. Uterine fibroid management: from the present to the future. Hum Reprod Update. 2016;

27. Luyckx M, Squifflet J-L, Jadoul P, Votino R, Dolmans M-M, Donnez J. First series of 18 pregnancies after ulipristal acetate treatment for uterine fibroids. Fertil Steril. 2014;102
28. Czuczwar P, Woźniak S, Szkodziak P, Wozniakowska E, Paszkowski M, Wrona $W$, et al. Predicting the results of uterine artery embolization: correlation between initial intramural fibroid volume and percentage volume decrease. Prz Menopauzalny. 2014;13

29. Fauser BCJM, Donnez J, Bouchard P, Barlow DH, Vazquez F, Arriagada P, et al. Safety after extended repeated use of ulipristal acetate for uterine fibroids. PLoS One. 2017;12

\section{Ready to submit your research? Choose BMC and benefit from:}

- fast, convenient online submission

- thorough peer review by experienced researchers in your field

- rapid publication on acceptance

- support for research data, including large and complex data types

- gold Open Access which fosters wider collaboration and increased citations

- maximum visibility for your research: over $100 \mathrm{M}$ website views per year

At BMC, research is always in progress.

Learn more biomedcentral.com/submissions 